\title{
La teoría de la materia de la Naturphilosophie*
}

The Theory of Matter of Naturphilosophie

Violeta Aréchiga ${ }^{\dagger}$

\section{Resumen}

El objetivo de este trabajo es subrayar que la teoría de la materia de la Naturphilosophie, tal y como se presenta en algunos textos de Schelling, tiene entre sus motivaciones el responder al reto planteado por la filosofía kantiana: el problema de los límites de nuestro conocimiento, ejemplificado por la biología y por la cuestión de la epigénesis. Dicha teoría de la materia forma parte de una discusión mucho más amplia en la que Schelling, ambiciosamente, intenta mostrar la identidad entre nuestro espíritu y la naturaleza. Esto lo conduce a desarrollar una visión de la naturaleza que podemos llamar organicista, en la medida en que, por un lado, es el organismo el que proporciona el modelo para pensarla y que, por otro, la actividad propia de la naturaleza es entendida en gran medida en términos de epigénesis.

Palabras clave: teoría de la materia - Naturphilosophie - organismo - epigénesis - teleología

\begin{abstract}
The aim of this text is to underline that Schelling's Naturphilosophie has among its motivations to respond to the challenge posed by the Kantian philosophy: the problem of the limits of our knowledge, exemplified by biology and the question of epigenesis. This theory of matter is part of a broader discussion in which Schelling ambitiously tries to show the identity between our spirit and nature. As a consequence, Schelling develops a vision of nature that we may call organicist, to the extent that, firstly, the body provides the model to think it and, secondly, the activity of nature is understood largely in terms of epigenesis.
\end{abstract}

Keywords: theory of matter - Naturphilosophie - organism - epigenesis - teleology

\footnotetext{
* Recibido: 1 de Junio de 2014. Aceptado en versión revisada: 22 de Agosto de 2014.

† Universidad Autónoma Metropolitana, Cuajimalpa, México. Para contactar a la autora, por favor, escribir a: violetare@gmail.com. Metatheoria 5(1)(2014): 7-20. ISSN 1853-2322.

(c) Editorial de la Universidad Nacional de Tres de Febrero. Publicado en la República Argentina.
} 


\section{Introducción}

The time has come for a reappraisal of Naturphilosophie [...]. Re-evaluating real Naturphilosophie could lead to a critique of our analytical, reductionistic, anti-holistic conception of natural science (Huneman 2007, pp. 17-18).

De acuerdo con Keith R. Peterson (2004), la Naturphilosophie se ha definido, negativamente, como la crítica de la ciencia mecanicista y determinista; como un punto de vista para el cual el reduccionismo del mecanicismo ilustrado constituía una traición a la complejidad de la naturaleza. Del lado positivo se ha dicho que la Naturphilosophie se centra en una visión dinámica, orgánica y holista del mundo natural en la que los seres humanos son integrados al mundo en lugar de ser separados de él. Es debido a este tipo de características que resulta importante actualmente volver nuestra mirada a la filosofía natural alemana de finales del siglo XVIII y principios del XIX y es que:

resulta claro, si prestamos atención a las tendencias recientes en biología evolutiva y del desarrollo, cosmología, ecología, teoría crítica y estudios de la ciencia, que muchos de los métodos críticos y aspiraciones programáticas de la filosofía de la naturaleza clásica están hoy nuevamente en ascenso (Peterson 2004, p. 8).

Cabe destacar en este sentido que uno de esos aspectos programáticos involucraba una nueva formulación de la relación entre los seres humanos y la naturaleza, una nueva manera de entender juntas a la epistemología y la ontología, que puede ser sumamente importante para la filosofía de la biología contemporánea. Asimismo, el punto de vista holista de Schelling y la Naturphilosophie es relevante para el intento contemporáneo, en ese terreno, de cuestionar el reduccionismo. Pero además, y esto también tiene valor en la reflexión filosófica e histórica acerca de la biología, la reconsideración y revaloración de la Naturphilosophie y de los puntos de vista de Schelling nos ha permitido ver que ambos jugaron un papel fundamental en el nacimiento de la biología. Se puede argumentar que prestar atención a la filosofía de Schelling es relevante historiográficamente porque:

La formalización de la biología como ciencia autónoma a principios del siglo XIX implicó el giro desde una comprensión regulativa de la teleología a una constitutiva, giro que recibió el mayor apoyo de la Naturphilosophie de Schelling. La biología como ciencia se hizo posible sólo cuando la organización comenzó a considerarse como un carácter constitutivo de los seres vivos que, como tal, requiere de explicación científica (Gambarotto 2014, p. 140).

La biología de Treviranus fue resultado de un proceso conceptual cuyo interés era proporcionar una definición y un marco explicativo de la manera en que la naturaleza viva se autoorganiza. Ese proceso, de acuerdo con Gambarotto, comenzó con el debate Haller-Wolff en el siglo XVIII y culminó con la idea de Schelling de una naturaleza como sistema dinámico capaz de autoorganizarse. Es posible afirmar entonces que la filosofía de Schelling reviste importancia, hoy en día, por razones que son tanto de orden filosófico-biológico como historiográfico.

El objetivo de este trabajo es poner de relieve que la teoría de la materia de la Naturphilosophie, tal y como se presenta en algunos textos de Schelling, tiene entre sus motivaciones el responder al reto planteado por la filosofía kantiana: el problema de los límites de nuestro conocimiento, ejemplificado por la biología y por la cuestión de la epigénesis. Dicha teoría de la materia forma parte de una discusión mucho más amplia en la que Schelling, ambiciosamente, intenta mostrar la identidad entre nuestro espíritu y el mundo natural. Esto lo conduce a desarrollar una visión de la naturaleza que podemos llamar con justicia organicista, en la medida en que, por un lado, es el organismo el que proporciona el modelo para pensarla y que, por otro, la actividad propia de la naturaleza es entendida en gran medida en términos de epigénesis. Tras la concepción dinámica de la materia propuesta por Schelling encontramos la intención de dar cuenta de la vida de una manera que sin ser mecanicista 
tampoco es vitalista y en la que la finalidad inherente al organismo proviene de su interior en virtud de un movimiento interno, constante, intencional, de fuerzas en continua oposición.

A fin de mostrar esto, en la primera parte presento una breve discusión del problema de la epigénesis tal y como se planteaba a finales del siglo XVIII. Expondré ahí algunas de las ideas de Blumenbach a este respecto, en particular su propuesta de una Bildungstrieb; la forma en que Herder apoyaba la alternativa epigenetista y la concepción kantiana de organismo que niega, en última instancia, que la biología pueda ser realmente una ciencia.

A continuación, en la segunda parte, intentaré delinear la forma en que Schelling parte de la concepción kantiana de organismo para tender puentes entre la naturaleza y el espíritu y presentar una concepción de la naturaleza, y en especial de la materia y las fuerzas, que constituyen a esa naturaleza, en la que, al igual que en la epigénesis, el carácter con arreglo a fines aparece como sobresaliente. Estamos ante una propuesta claramente teleológica, pero en la que la teleología aparece no como algo proveniente desde fuera de la naturaleza sino como interno a ella.

\section{Kant, Herder y la epigénesis}

Como sabemos, a fines del siglo XVIII encontramos todavía una fuerte discusión en torno al problema de la generación -discusión cuyos inicios se remontan a mediados del siglo XVII y que se sitúa en el marco de la filosofía mecanicista-. Las dos visiones que se debatían eran la teoría de la preformación de los gérmenes y la de la epigénesis. Para la primera, el desarrollo del embrión era una suerte de despliegue de estructuras ya preexistentes, contenidas en el óvulo o en el espermatozoide; ahí donde el mecanicismo no alcanzaba a explicar, basándose sólo en la materia y su movimiento, de qué manera un ser vivo era capaz de dar lugar a otro semejante a partir de una materia sin forma, la preexistencia o la preformación del germen aparecía como una solución casi natural. En contraste, las teorías de la epigénesis a las que se oponía el preformacionismo sostenían que a partir de materia sin forma o sin estructura se originaba un nuevo organismo, se desarrollaba un embrión, en virtud de la acción de fuerzas vitales inaceptables para la filosofía mecanicista.

La epigénesis [...] argumentaba que cada embrión se produce de novo mediante un desarrollo gradual de materia no organizada. Se propusieron varias explicaciones acerca de cómo se lograba esta formación gradual, pero los epigenetistas coincidían en su oposición a la preexistencia (Roe 1981, p. 1).

Las disputas sobre la generación se habían avivado luego de la postulación, por parte de Buffon y Maupertuis, de una segunda versión de la teoría de la epigénesis en la que se intentaba explicar la formación del embrión apelando solamente a una concepción particulada de la materia, fuerzas de contacto y leyes del movimiento, sin hacer uso de fuerzas vitales. El problema residía en la aparente imposibilidad de explicar que la materia pudiera auto-organizarse con base en fuerzas propias, inmanentes, sin ninguna intervención divina. Haller, en 1752, sugirió que la cuestión podría resolverse mediante una teoría de fuerzas naturales que poseyeran una dirección teleológica innata capaz de organizar la materia orgánica. Aunque “durante mediados de los 50's, Haller continuó apoyando un recuento de la generación a través de la acción de fuerzas teleológicas constructivas" (Sloan 2002, p. 235), luego abandonaría esta postura a favor de una preformacionista. Por su parte, Blumenbach, tanto en su ensayo sobre el Bildungstrieb de 1780 como en la segunda edición y siguientes de su Manual de Historia Natural (A Manual of the Elements of Natural History [Blumenbach 1825]), presentó una fuerte defensa de la teoría de la epigénesis en la que se postuló el carácter teleológico de las fuerzas naturales que construyen al ser vivo.

"A fin de explicar la formación de los cuerpos organizados", nos dice Blumenbach $(1825$, p. 8), se ha propuesto la teoría de la preformación, pero de acuerdo con ella en realidad ningún ser vivo se genera, sino que ha existido desde la primera generación como un germen perfecto, preformado, al interior de sus ancestros. $Y$ añade: 
Es mucho más consonante con los poderes de nuestro entendimiento y con las reglas del estudio filosófico de la naturaleza, explicar el origen de los cuerpos organizados mediante la formación progresiva (epigénesis) de la materia seminal, en sí misma no organizada, pero susceptible de organización en ciertas circunstancias (Blumenbach 1825, p. 10).

Ahora bien, aclara, hay diversas maneras de explicar la formación progresiva. Por ejemplo, un tal Mazini postuló que la descendencia se formaba mediante un cierto tipo de cristalización, lo cual es también una especie de epigénesis. Este punto de vista, mecanicista, es para Blumenbach totalmente inadmisible en la medida en que supone que los cuerpos organizados se forman en virtud de una "vis plastica" que también prevalece en el reino mineral e ignora, y esta diferencia es fundamental, que los cuerpos organizados poseen siempre un carácter teleológico.

En contraste, Blumenbach propone que la materia seminal de los progenitores, madura ya y no organizada pero sí organizable, cuando se transmite en el momento adecuado y en ciertas circunstancias "cae bajo la influencia de un poder vital, el así llamado impulso formativo (nisus formativus, Bildungstrieb) que da origen a las acciones adecuadas" (Blumenbach 1825, p. 11). Se trata de un impulso que difiere de poderes formativos mecánicos como el de la cristalización en la medida en que es capaz de darle forma a la materia seminal organizable de modos que se correspondan con los propósitos o fines que los cuerpos organizados deben cumplir. El impulso formativo es pues un principio teleológico y, combinado con el mecánico, produce en primer lugar la formación progresiva a partir del momento de la concepción; en segundo lugar, el mantenimiento del ser vivo, o la nutrición; y, en tercer lugar, la reparación, en la medida de lo posible, de los daños accidentales que el ser en cuestión pueda llegar a sufrir. El término impulso formativo, reconoce Blumenbach haciendo un gesto a la retórica newtoniana,

Como los nombres aplicados a cualquier otra clase de poder vital, en sí mismo no explica nada; sirve únicamente para designar un poder peculiar formado por la combinación del principio mecánico con el que es susceptible de modificación; un poder cuya actividad constante establecemos por experiencia, mientras que su causa, como la de todos los otros poderes naturales generalmente reconocidos, sigue siendo en el sentido más estricto de la palabra "qualitas occulta" (Blumenbach 1825, p. 12).

Finalmente, el impulso formativo, al actuar de una forma determinada y sobre un objeto en particular, en materiales capaces de ser influidos y organizados por él, conserva "la forma y el hábito determinados de todas las especies individuales de seres vivientes” (Blumenbach 1825, p. 12).

Pues bien, es en este contexto de debate en torno a la generación de los organismos que tiene lugar una parte importante del trabajo de Kant. De acuerdo con Sloan (2002) el encuentro de Kant con las ciencias de la vida de la época nos ayuda a comprender la formulación del programa filosófico maduro de este autor. Específicamente, según el mismo Sloan, Kant habría enfrentado seriamente el tema de la epigénesis a raíz de su lectura del texto de Herder de 1784 Ideas para una filosofía de la historia de la humanidad.

Herder adopta en este texto una postura decididamente anti-preformacionista. "La teoría de los gérmenes", dice, "no explica en realidad nada: pues el germen ya es una forma, y donde hay una forma debe haber habido un poder orgánico que la formara" (Herder [1784] 1800, p. 51). Nadie ha visto gérmenes preformados, declara, y sí en cambio podemos ver en la existencia de un ser vivo poderes orgánicos actuando; por ejemplo, un individuo que posee este tipo de poderes puede propagar su especie sin más ayuda. Ahora bien, en la medida en que una criatura es más compleja su germen resulta ser más difícil de distinguir, así que podríamos decir que una criatura de organización compleja "es materia orgánica, que debe recibir poderes vitales antes de que pueda alcanzar la forma de la criatura futura" (Herder 1800, p. 111).

Los poderes orgánicos deben destruir mientras arreglan; atraer entre sí partes y separarlas; pareciera como si varios poderes se hallaran en lucha y fueran al principio a producir un aborto, hasta que un equilibrio se establece entre ellos y la criatura deviene lo que debía ser siguiendo su linaje.

Es para la formación (génesis), un efecto de poderes internos, para lo cual la naturaleza ha preparado una masa a la que ellos han de dar forma, y en la que se han de desplegar. Esto es lo que la experiencia 
de la naturaleza, los periodos de formación en las distintas especies de acuerdo con su mayor o menor organización compleja y totalidad de poder vital, confirman (Herder 1800).

Así pues, claramente, para Herder podía prescindirse de los gérmenes preformados y poner en su lugar una materia orgánica sobre que la actúan fuerzas vivientes para producir la organización. Pero a los ojos de Kant el texto de Herder mostraba los excesos a los que podía llegar una presunción epistemológica realista sin restricciones acerca de la cognoscibilidad de la historia de la naturaleza (Sloan 2002, p. 243).

En la Crítica del Juicio Kant había definido un organismo, un propósito natural como lo llama, como algo que es la vez causa y efecto de sí mismo. En el ejemplo que nos proporciona, el de un árbol, éste se produce a sí mismo en el sentido de que produce a otro ser de su misma especie. Es, en este sentido, tanto causa como efecto al interior de su especie. Además se produce a sí mismo en tanto individuo.

En primer lugar, un árbol genera otro árbol de acuerdo con una ley natural familiar. Pero el árbol que produce es de la misma especie. Por tanto, con respecto a su especie el árbol se produce a sí mismo: al interior de su especie, es tanto causa como efecto, tanto al generarse a sí mismo como al ser generado por sí mismo incesantemente, preservándose en consecuencia a sí mismo como especie. (Kant 1987, p. 249)

Un ser vivo es aquél en que una parte produce a la otra y que es capaz de producir a otro ser vivo, remplazar sus propias partes, compensar la falta de alguna de sus partes con ayuda de las otras y repararse a sí mismo.

Un ser organizado no es una mera máquina. Pues una máquina posee sólo fuerza motora. Pero un ser organizado posee en su interior una fuerza formativa, y una fuerza formativa que este ser imparte a las clases de materia que carecen de ella (organizándolas de este modo). Esta fuerza es por tanto una fuerza formativa que se propaga a sí misma -una fuerza que la mera habilidad [de una cosa] para mover [otra] (es decir, el mecanismo) no puede explicar (Kant 1987, p. 253).

Cuando Kant nos habla de fuerza formativa está retomando las ideas de Blumenbach y, como él, se niega a reducir dicha fuerza a una concepción meramente mecánica. La fuerza formativa, para Kant, no puede ser explicada por el puro mecanismo. Al contrario, sostiene, la naturaleza se organiza a sí misma y lo hace en cada especie de sus productos organizados.

Pero una vez llegados a este punto se presenta un problema. Y es que si pensamos en esa propiedad organizadora como un "análogo de la vida" estaríamos dotando a la materia con la propiedad de la vida, como hace el hilozoísmo, o bien tendríamos que apelar a un principio ajeno, un alma, que se une a la materia y ambas cosas son, a los ojos de Kant, inaceptables (Kant 1987, p. 254). Así que,
Estrictamente hablando [...] la organización de la naturaleza no posee nada análogo a ninguna causalidad conocida por nosotros. [...] El concepto de una cosa como un propósito natural en sí mismo no es un concepto constitutivo ni del entendimiento ni de la razón. Sin embargo, puede ser todavía un concepto regulativo para el juicio reflexivo, permitiéndonos usar una analogía remota con nuestra propia causalidad en términos de propósitos en general, guiar nuestra investigación de los objetos organizados y meditar con respecto a su base suprema -una meditación con el propósito no de obtener conocimiento ni de la naturaleza ni de la base original de la naturaleza, sino con el propósito de [auxiliar] ese mismo poder práctico en nosotros [esto es, nuestra razón] por analogía con el cual considerábamos la causa del carácter con arreglo a fines de los objetos organizados (Kant 1987, p. 255).

Para Kant la biología no puede ser una ciencia y se comprende su reacción ante Herder. Éste había sostenido que incluso hoy todavía la naturaleza produce todas las cosas constantemente a partir de lo más pequeño: desde sus inicios, la naturaleza misma estableció los fundamentos de "la formación y evolución de las criaturas" (Herder 1800, p. 8), de acuerdo con los cuales una serie de poderes activos aunados a los elementos dieron lugar a la Tierra, y diferentes combinaciones de agua, aire y luz originaron las primeras semillas de los vegetales. Luego de que muchas plantas brotaran y murieran, 
nacieron los animales organizados; primero, insectos, aves, animales acuáticos y animales nocturnos; después los “animales más perfectos" y, por último, el ser humano (Herder 1800, p. 8).

La naturaleza [...] supo cómo estructurar una organización por medio de la cual dos criaturas se unen en una sola, y de su conjunción una tercera se forma, la estampa de ambos, en el instante de la tibieza orgánica vital más íntima.

Así concebido, solamente por este medio se cría el nuevo ser. El calor materno lo rodea y le da forma. Sus pulmones todavía no respiran [...] En la proporción en que su calor interno es avivado por el calor de la madre, su corazón deviene más perfecto y la sangre se enrojece, y adquiere una enérgica circulación, aunque todavía no puede entrar en contacto con los pulmones. Con un pulso claramente latiendo, la criatura se mueve; y al final llega al mundo perfectamente formada, dotada con todas las facultadas de percepción y movimiento voluntario (Herder 1800, pp. 46-47).

Para Herder, no sólo el feto se desarrolla gradualmente a partir de la materia sin forma; asimismo, el mundo se ha formado a lo largo del tiempo de manera epigenética. En ambos casos, además, encontramos una formación progresiva con arreglo a fines.

¿Qué está construido con más arte que la casa de un caracol? Las celdas de la abeja deben rendirse ante ella, la red de la oruga, el gusano de seda, deben ceder su lugar a esta flor artificial. ¿Y por qué medios la Naturaleza ha logrado esto? Mediante poderes orgánicos internos (Herder 1800, p. 60).

De tal suerte, si para Kant en 1787 la biología es el ejemplar, el caso particular, de lo que no puede ser ciencia -ahí donde el problema general es el de los límites de nuestro conocimiento- la epigénesis se muestra como el reto central, porque es justamente en este proceso donde resalta de manera más evidente la necesidad de postular una fuerza formativa y teleológica de la cual, para la filosofía kantiana, no podemos tener un conocimiento cierto: "Kant mantenía [...] que la biología no podía realmente ser una ciencia, sino en el mejor de los casos un laxo sistema de regularidades empíricas inciertas, no una Naturwissenschaft, sino una Naturlehre" (Richards 2002, p. 231).

\section{Schelling}

Schelling, por su parte, retomará a Herder y tratará se sortear las restricciones impuestas por Kant al conocimiento en general y a la biología en particular. Herder fue para Schelling una fuerte influencia y, junto con él, concibió a la naturaleza como "un organismo que se autoproduce y que por lo tanto consiste en su propia historia” (Leyte 1996, p. 29). Hasta el título de la obra de Schelling (1797), Introducción a ideas para una filosofía de la naturaleza, fue al parecer inspirado por el texto de Herder.

Tradicionalmente, la Naturphilosophie se ha asociado a una concepción organicista de la naturaleza. El organismo, entendido a la manera de Kant, se convierte en un "paradigma destinado a pensar el conjunto de los fenómenos de la naturaleza" (Lemaitre 2013, p. 3). Por otro lado, como en un eco de Herder, se intenta dar cuenta del paso de la naturaleza inorgánica a la orgánica en términos de una evolución progresiva.

La diferencia entre la naturaleza orgánica y la naturaleza inorgánica no se niega, pero se intenta pensar un pasaje, una evolución progresiva de la una a la otra, lo cual pasa por el reconocimiento, en los fenómenos físicos y químicos, de caracteres que, por así decirlo, anticipan lo viviente (Lemaitre 2013, p. $3)$.

Ante todo, la totalidad de la naturaleza se considera como un organismo y la relación entre el todo y las partes se piensa, en su mayor generalidad, desde el modelo de la organización. Por su parte el organismo, kantianamente entendido, posee una singularidad distintiva: la de su carácter con arreglo a fines. El mecanicismo, dirá Schelling, no constituye a la naturaleza -lo cual quiere decir que tampoco nos permite comprenderla- porque al considerar la naturaleza orgánica no encontramos vinculaciones mecánicas entre causas y efectos: "todo producto orgánico existe por sí mismo, su existencia no depende de ninguna otra” (Schelling 1797, p. 96). El organismo se produce a sí mismo y surge de sí mismo, dice 
como parafraseando a Kant, y además todos los organismos singulares producen y reproducen su propio género hasta el infinito.

Así pues, un organismo como tal no es ni causa ni efecto de una cosa exterior a él y por lo tanto no es nada que se entrometa en la conexión del mecanicismo. Todo producto orgánico lleva el fundamento de su existencia dentro de sí mismo, porque es causa y efecto de sí mismo (Schelling 1797, p. 97).

A diferencia de Kant, sin embargo, para Schelling entre las partes y el todo existe una relación objetiva de modo tal que a todo organismo "subyace un concepto", pero un concepto que reside al interior del organismo mismo y que no puede separarse de éste.

En su texto de 2013 Lemaitre señala que se considera a Schelling como uno de los arquitectos de la Naturphilosophie y el que le proporciona una justificación y una sistematización propiamente filosóficas. Esto lleva a Lemaitre a preguntarse por el lugar preciso que este autor le atribuye a las nociones de organismo y de organización en su filosofía de la naturaleza y de entrada responde que el concepto de organismo no es el único concepto central de la filosofía de la naturaleza de Schelling; que una de sus inspiraciones más importantes es una concepción dinámica de la materia heredada en última instancia de Kant. En efecto, en Introducción a ideas para una filosofía de la naturaleza Schelling trata en la primera parte cuestiones de física y química y no de la naturaleza orgánica, y en la segunda del proyecto de un sistema de la ciencia de la naturaleza.

Pero en la Introducción al proyecto... (Schelling 1799), como reconoce Lemaitre, el estudio de la naturaleza orgánica sí se hace presente, junto con proyecto general de una filosofía de la naturaleza que se introduce a propósito del problema planteado por Kant, el de la realidad de nuestro conocimiento. La solución propuesta por Schelling es atrevida:

No consideramos el sistema de nuestras representaciones en su ser, sino en su devenir. La filosofía se torna genética [...]. A partir de aquí ya no es posible ninguna separación entre experiencia y especulación. El sistema de la naturaleza es al mismo tiempo el sistema de nuestro espíritu (Schelling 1797, pp. 95-96).

Existe, en otras palabras, una identidad entre el sistema del espíritu y el sistema de naturaleza. Con el fin de mostrar esto, Schelling hace uso del modelo orgánico: el organismo es la imagen o el análogo del espíritu en la medida en que posee una actividad espontánea y una fuerza interna mediante la cual se produce a sí mismo. Del mismo modo, la configuración con arreglo a fines del organismo es un signo de la homogeneidad entre el espíritu y la naturaleza.

Para Schelling, [la] vinculación con arreglo a fines de la materia y la forma no puede ser sino de naturaleza ideal, en el sentido de que no es sino para un espíritu que una vinculación así puede existir. Esto no significa, sin embargo, que ésta no sea más que una proyección subjetiva, sino más bien que la objetividad misma que uno está obligado a reconocer en la configuración con arreglo a fines de los productos orgánicos - pues estos productos existen en efecto independientemente de nosotros en la naturaleza- es el signo de una homogeneidad originaria entre el espíritu y la naturaleza (Lemaitre 2013, pp. 19-20).

Los productos orgánicos poseen una finalidad respecto a la cual el mecanicismo no tiene nada que decir. Una vez más Schelling insiste en que "de nada nos sirve ya aquí separar como bien nos plazca concepto y objeto, forma y materia. Porque, por lo menos, aquí ambas cosas se encuentran originaria y necesariamente unidas" (Schelling 1797, pp. 97-98). Pero esa unidad no se puede explicar a partir de la materia como tal, porque la finalidad sólo puede comprenderse en relación con un entendimiento que juzga. Tampoco es posible suponer que la finalidad es imbuida desde fuera por Dios, pues esto es reducirlo a mero "arquitecto de la naturaleza". Así que, según Schelling, no nos queda más camino que asumir que la vida, de algún modo, está presente en toda la naturaleza: "Hasta en la materia meramente organizada hay vida, aunque sea vida de un tipo muy limitado" (Schelling 1797, p. 102).

Esta idea es tan antigua y se ha mantenido hasta nuestros días de manera tan firme, bajo las más diversas formas (ya en los tiempos antiguos se admitía que el mundo entero estaba penetrado por un principio que lo animaba, al que se llamaba alma del mundo [...]), que seguramente es lícito presumir de 
antemano que tiene que residir algún fundamento para esta creencia natural en el espíritu humano (Schelling 1797, p. 102).

Ahora bien: los órganos animales poseen capacidades propias de lo vivo tales como la sensibilidad y la irritabilidad; sin embargo, esas capacidades no son suficientes para explicar la vida. Para ello se requiere de "un principio más elevado al que no podemos explicar a partir de la propia materia" (Schelling 1996, p. 104). Puede considerarse al cuerpo a la manera cartesiana como una suma de partículas organizadas, una máquina hidráulica o hasta un laboratorio químico -pero todo ello deja sin responder la pregunta de cuál es el principio que agrupa orgánicamente todos los cambios en el cuerpo.

Una primera respuesta a dicha pregunta podría ser que en el cuerpo vivo reside necesariamente un principio que le permite sustraerse a las leyes de la química y que tal principio es una fuerza vital. No obstante, Schelling rechaza esta posibilidad porque, sostiene, una fuerza sólo puede pensarse como algo finito y ninguna fuerza es finita por sí misma; para serlo, tiene que estar limitada por otra fuerza opuesta. Entre esas dos fuerzas o existe un equilibrio relativo -pero esto las haría extinguirse- o se encuentran en conflicto permanente. En este último caso sería necesario un tercer factor que mantuviera a las fuerzas en ese conflicto, pero "dicho tercero no puede ser también una fuerza, pues de lo contrario volveríamos a caer en la precedente alternativa” (Schelling 1797, p. 105). Es evidente entonces, concluye Schelling, que el concepto de fuerza vital es inútil.

Hay una salida a este problema que consiste en partir de la consideración de que "la primera máxima de toda verdadera ciencia de la naturaleza [es] explicar todo a partir de las fuerzas de la naturaleza" (Schelling 1797, p. 121). En otras palabras, a fin de resolver la cuestión de la organización de los seres vivos Schelling acudirá a lo que en su texto de 1799 llama "física especulativa".

Para instaurar verdaderamente una física especulativa sólo nos queda un camino, el dinámico, con el presupuesto de que el movimiento no sólo surja del movimiento sino también del reposo -y por lo tanto que también en el reposo de la naturaleza esté implícito el movmiento- y de que todo movimiento mecánico sólo sea un movimiento secundario y derivado de ese movimiento primitivo y orignario. (Schelling 1797, p. 123)

La física especulativa se ocupará de las causas originarias del movimiento en la naturaleza o, en otras palabras, de las manifestaciones dinámicas. Se trata de una física especulativa que se orienta a lo que nuestro autor llama aquí "el impulso interno" y lo "no objetivo de la naturaleza" (a diferencia de una física empírica que se queda meramente en la superficie) y que pretende abarcar las manifestaciones dinámicas en su conjunto - manifestaciones que dan lugar no a un mundo sino a una naturaleza, es decir, no a un producto sino a algo que es a la vez productivo.

Esta oscilación de la naturaleza entre la productividad y el producto tendrá que manifestarse como una duplicidad general de los principios por medio de la cual la naturaleza se mantiene en constante actividad evitando de ese modo agotarse en su propio producto; dicha dualidad general, como principio de toda explicación de la naturaleza, será tan necesaria como el propio concepto de la naturaleza. (Schelling 1797, p. 125).

La naturaleza es para nuestro autor un todo orgánico, viviente y activo por sí mismo y es justamente en este sentido al menos que la vida se hace presente a todo lo largo y ancho de la naturaleza: la "identidad de productividad y producto del concepto originario de naturaleza se expresa en las concepciones habituales de la naturaleza como un todo que por sí mismo es simultáneamente causa y efecto" (Schelling 1797, pp. 131-132). Incluso las "manifestaciones" magnéticas y eléctricas no son solamente efecto de una cierta materia sino transformaciones en el modo de subsistir de una materia que a su vez depende de la interacción continua de fuerzas fundamentales.

Sin embargo, la identidad productividad-producto de la naturaleza no se presenta de forma inmediata. Al contrario, es un resultado de un proceso en el que la naturaleza deviene primero objeto; se transforma desde el puro sujeto, o productividad, en objeto, producto, y esto, dice Schelling, es impensable si la naturaleza no se dividiera originalmente. Hay aquí una duplicidad originaria en la 
que, justamente desde el inicio, residen tendencias contrapuestas. Sin embargo, para constituir el producto esas tendencias opuestas deben necesariamente coincidir y en tal coincidencia se aniquilan.

\begin{abstract}
Esta contradicción inevitable [...], que el producto sólo puede surgir gracias al concurso de tendencias opuestas, pero que dichas tendencias opuestas se anulan mutuamente, sólo puede resolverse de la siguiente manera: resulta absolutamente impensable la permanencia de un producto sin una permanente reproducción. Hay que prensar el producto como constantemente aniquilado y constantemente reproducido de nuevo (Schelling 1797, pp. 135-136).
\end{abstract}

Por lo tanto, lo que percibimos en la naturaleza no es el producto originario sino su evolución, su formación constante y la condición de toda formación es, para Schelling, la dualidad. De ahí que, añade, la materia en Kant se construya a partir de fuerzas opuestas.

Esta dualidad, duplicidad, tiene su esquema en los fenómenos eléctricos, en lo negativo y lo positivo; estos son el esquema general para la construcción de la materia en general. Es en este mismo sentido que Schelling afirma que la productividad, la actividad constante de la naturaleza, requiere en primer lugar de una escisión:

Lo primero que se exige es una escisión de la productividad [...]. La escisión seguramente determina una alternancia de contracción y expansión. Esta alternancia no es algo que se encuentre en la materia, sino la materia misma y el primer grado de la productividad en su paso hacia el producto (Schelling 1797, p. 148).

Pareciera pues que en el seno de esta naturaleza dinámica la materia misma es activa, contrayéndose y expandiéndose continuamente en una interacción de las fuerzas de atracción y repulsión a las que autores como Newton, Hales y Buffon, habían apelado anteriormente. Y hay más todavía, porque "en las direcciones opuestas que surgen por medio de esa oposición reside el principio para la construcción de todas las manifestaciones de la vida" (Schelling 1797, p. 150).

La productividad, continúa Schelling, debe estar limitada originariamente y esa limitación es resultado de una oposición que surge al interior de ella misma. "Esta diferencia, pensada puramente, es la primera condición de toda actividad de la naturaleza; la productividad se encuentra atraída y repelida entre opuestos" (Schelling 1797, p. 153). Y nuevamente señala que los fenómenos eléctricos son el esquema de esa naturaleza que oscila entre productividad y producto. Ese estado de oscilación, declara, de alternancia entre una fuerza de atracción y otra de repulsión, es "el auténtico estado de la formación" (Schelling 1797, p. 153).

Y es que a Schelling en realidad no le interesa dar cuenta del mundo inorgánico, "un mundo que no es productivo", sino para poder cuenta del mundo productivo, la naturaleza orgánica. Sin embargo, sólo podemos conocer esta última por oposición al primero. Por tanto, "la tarea más general de la física especulativa se deja formular ahora del siguiente modo: conducir a una expresión común la construcción de productos orgánicos e inorgánicos" (Schelling 1797, p. 151).

Con respecto a este objetivo general, nuestro autor afirma que los fenómenos que se entienden bajo el nombre de proceso dinámico, los fenómenos verdaderamente primitivos de la naturaleza, son solamente la permanente autoconstrucción de la materia. Pero para cualquier construcción se requiere una oposición originaria de fuerzas: una fuerza expansiva que tiende al exterior y una fuerza atractiva, que tiende hacia el interior. Del carácter continuo y omnipresente de esa oposición tenemos muchos ejemplos, entre ellos el de la aguja magnética que nos muestra una "permanente y renovada anulación de la indiferencia” en su movimiento hacia los polos. En todo caso, podemos distinguir tres grados, una verdadera progresión, en el proceso dinámico: el magnetismo, la electricidad y el proceso químico. "La última síntesis de la materia termina en el proceso químico" (Schelling 1797, p. 162). En esos tres grados encontramos las categorías originarias de construcción de una naturaleza totalmente ajena al reposo, pero también ajena a cualquier tipo de fuerza o influencia proveniente desde su exterior.

Así como en la naturaleza orgánica está presente en cada individuo -en la gradación de la sensibilidad, la irritabilidad y el impulso hacia la reproducción - el secreto de la producción de toda la naturaleza orgánica, del mismo modo, en la sucesión gradual del magnetismo, la electricidad y el proceso químico 
-la cual es distinguible en los cuerpos singulares- está presente el secreto de la producción de la naturaleza a partir de sí misma (Schelling 1797, pp. 165-166).

Y es en estas categorías donde reside finalmente la explicación, por un lado, de la riqueza y diversidad tanto de la naturaleza orgánica como la inorgánica y, por otro, de la relación entre lo inerte y lo propiamente vivo. La naturaleza orgánica y la inorgánica tienen en común el que ambas transforman, hasta el infinito, las relaciones entre magnetismo, electricidad y proceso químico. Sin embargo, mientras que la naturaleza inorgánica comienza a partir de "factores simples", la naturaleza orgánica comienza a partir de productos que, a su vez, se convierten en factores. Y en este marco la vida consiste en impedir constantemente que la productividad termine definitivamente en producto; la vida se renueva permanentemente a sí misma.

Por otro lado, se puede decir también, en otro sentido, que la construcción del producto orgánico y el inorgánico no se puede llevar a una expresión común.

La tarea presupone que el producto orgánico y el producto inorgánico se encuentran opuestos cuando la verdad es que el orgánico sólo es la potencia superior del inorgánico y resulta de la potencia superior de esas mismas fuerzas que también producen al inorgánico. La sensibilidad sólo es la potencia superior del magnetismo, la irritabilidad sólo la potencia superior de la electricidad, el impulso reproductivo sólo la potencia superior del proceso químico (Schelling 1797, p. 169).

Lo que resulta claro en esta concepción organicista de la naturaleza propuesta por Schelling es que no existe un abismo insalvable entre lo vivo y lo muerto. Al contrario, el hecho de mirar lo inorgánico a través del lente proporcionado por los seres vivos, organizados, conduce a nuestro autor a adjudicarle un carácter dinámico a la materia inorgánica que, gracias a esa misma dinamicidad, impide la explicación de la naturaleza viviente en términos mecánicos o providencialistas.

Schelling afirma que "la existencia de la materia se basa en la oposición de dos fuerzas" (Schelling 1797, p. 198), la fuerza de atracción y la de repulsión, pero que lo que hace posible la materia no es una relación de mero concurso entre esas fuerzas, sino su comportamiento recíproco en relación con el espacio. Esta concepción dinámica de la materia, me parece, no es solamente una perspectiva inspirada por la de Kant y más lejanamente por la de Newton ${ }^{1}$ y varios de sus seguidores, sino un intento de dar respuesta a tres problemas: el de la epigénesis, el de la biología en tanto ciencia y del conocimiento en general.

De acuerdo con Lemaitre la primera parte del texto de Schelling de 1798, El alma del mundo (Von der Weltseele), expone la fundamentación de la filosofía de la naturaleza a partir de una concepción dinámica de la materia marcada por el modelo de la polaridad. De manera semejante a la de los textos que he mencionado aquí, en éste la materia se considera producto de una dualidad de fuerzas o principios opuestos, en cuyo interior uno de ellos juega el rol de un principio positivo y el otro de un principio negativo. En ese contexto, el de una teoría de la materia propiamente dicha, señala Lemaitre que no es el organismo el que proporciona el paradigma principal, sino la electricidad y el magnetismo.

Si la segunda parte de El alma del mundo está dedicada a la ciencia del organismo, toda la primera parte, que se titula "Sobre la primera fuerza de la naturaleza" expone la fundamentación de la filosofía de la naturaleza sobre una concepción dinámica de la materia marcada por un modelo de polaridad. [...] En este contexto, no es el organismo quien de manera prioritaria proporciona el paradigma principal, sino más bien la electricidad y el magnetismo (Lemaitre 2013, p. 27).

Sin embargo, en varios pasajes de textos escritos por Schelling entre 1797 y 1800 encontramos lo que desde mi punto de vista es el motivo principal que impulsa su concepción dinámica de la materia: la posibilidad de dar cuenta de la vida, de lo orgánico y lo organizado, de una manera que no fuera mecanicista ni estrictamente hablando vitalista.

\footnotetext{
${ }^{1}$ En efecto, Newton decía en su Óptica: "Have not the small Particles of Bodies certain Powers, Virtues, or Forces, by which they act at a distance [...] upon one another for producing a great Part of the Phaenomena of Nature?” (Newton 1979, p. 375).
} 
Por supuesto Schelling rechaza de entrada la postura mecanicista. El mecanicismo no constituye la naturaleza, afirma, porque en el terreno de la naturaleza orgánica no hay una vinculación lineal, mecánica, de causas y efectos.

El mecanicismo no es, ni mucho menos, lo que constituye a la naturaleza. Porque cuando entramos en el reino de la naturaleza orgánica, cesa para nosotros toda vinculación mecánica entre causas y efectos. Todo producto orgánico existe por sí mismo, su existencia no depende de ninguna otra (Schelling 1797, p. 96).

Los productos orgánicos existen por sí mismos; el organismo se produce a sí mismo y surge de sí mismo. En ese sentido el organismo no es ni causa ni efecto de algo exterior a él. Ninguna de sus partes puede surgir sino al interior del todo, y ese todo consiste en la relación recíproca entre esas partes. Y "no sólo su forma, sino su existencia tienen una finalidad. El organismo no podría organizarse sin estar ya organizado” (Schelling 1797, p. 97). Se trata aquí de una finalidad interna, que no se impone desde fuera sino que surge, por decirlo así, al interior de la existencia misma del organismo. Pero si esto es cierto, el organismo es un objeto que subsiste por sí mismo, un todo en el que la forma es inseparable de la materia y que por tanto no puede explicarse mecánicamente, como tampoco puede explicarse mecánicamente el origen de la materia.

Schelling rechaza asimismo en este punto la posibilidad de la intervención divina. Los productos orgánicos poseen finalidad y ésta sólo se puede concebir en relación con un entendimiento que juzga, pero la finalidad propia de los productos naturales "habita en ellos mismos, [...] es objetiva y real y por lo tanto no pertenece a nuestras representaciones arbitrarias, sino a las necesarias" (Schelling 1797, p. 99). Suponer que gracias al poder creador de Dios ha surgido todo el sistema de la naturaleza no nos permite avanzar ni un paso. Y es que otorgarle este rol a Dios es permitir que la finalidad entre en la naturaleza como algo exterior a ella y "destruiréis de raíz toda idea de naturaleza en cuanto dejéis que la finalidad entre en ella desde fuera" (Schelling 1797, p. 101).

La Naturphilosophie se ve obligada a asumir que hasta en la materia meramente organizada está implícita ya alguna forma de vida. Ahora bien: para que la vida surja "hace falta un principio más elevado al que no podemos explicar a partir de la propia materia" (Schelling 1797, p. 104). No se puede considerar al cuerpo un mero agregado mecánico de partículas, porque, como decíamos antes, en esta consideración sigue persistiendo el problema de en qué forma se producen los cambios internos y, ante todo, de qué principio agrupa armónicamente esos cambios.

Así las cosas, la solución a este problema es la concepción dinámica de materia, esto es, esa concepción en la que el magnetismo, la electricidad y el proceso químico -cuya manera de ser es la polaridad-son funciones de la materia en general. Y, como también mencionamos ya, en este terreno el producto orgánico es la potencia superior del producto inorgánico, pero resultado de las mismas fuerzas que producen éste. Si la naturaleza orgánica sólo es la materia inorgánica repitiéndose en la "potencia superior", ello significa - para Schelling- que con las categorías para la construcción de la materia en general tenemos ya las categorías necesarias para la construcción del producto orgánico. En otras palabras, "si los fenómenos dinámicos son sólo manifestaciones de la naturaleza que se repite a sí misma en diferentes grados, entonces ya se ha dado con ellos el impulso hacia la naturaleza orgánica" (Schelling 1799, p. 219).

Para Schelling, es la concepción dinámica de la materia la que permite dar cuenta de la vida y su organización. Vimos que este autor declara que la Naturphilosophie debe asumir que hay una gradación de vida en la naturaleza y que incluso en la materia "meramente organizada" hay ya vida. Como si se tratara de una prueba, añade que la antigüedad de esta idea -conceptualizada como el alma del mundo- pareciera ser una creencia perfectamente natural para el ser humano.

El misterio que rodea el problema del origen de los cuerpos organizados procede, según Schelling, de que en esos seres se unen de manera íntima la necesidad y la contingencia; la necesidad porque la existencia misma de los cuerpos organizados es final, y la contingencia porque esa finalidad sólo puede devenir real para un ser con intuición y reflexión. "Por eso", nos explica, "el espíritu humano fue conducido tempranamente a la idea de una materia que se organiza a sí misma y-como la organización 
sólo es concebible en relación con un espíritu-, a la de la unión originaria del espíritu y la materia en estas cosas" (Schelling 1797, p. 102). Así que, por un lado, en los seres vivos tenemos una unidad originaria entre el espíritu y la materia. Por otro, como también dijimos, para Schelling un organismo no podría organizarse sin estar ya organizado. Y la "organización previa" de la que aquí habla este autor proviene en última instancia del carácter dinámico de las fuerzas que constituyen a la naturaleza porque, al interior de la identidad absoluta de la duplicidad de la naturaleza, surge una oposición y, a partir de ésta, surgen direcciones opuestas en las "reside el principio para la construcción de las manifestaciones de la vida" (Schelling 1799, p. 150).

\section{Conclusión}

Cuando Kant leyó a Herder se vio obligado a reconsiderar su postura preformacionista (cf. Sloan 2002), pero no su postura con respecto a los límites del conocimiento -como es evidente por sus afirmaciones en la Crítica del juicio-.

En 1785 Kant empleó, por primera vez, en sus escritos publicados el término embriológico "epigénesis". [...] Kant se había visto obligado a enfrentar [la] forma dinámica, vitalista de epigénesis en profundidad filosófica en virtud del uso de esta forma de epigénesis por parte de Johann Herder (Sloan 2002, p. 242).

Cuando, poco después, se encontró con Blumenbach, las ideas de éste tuvieron sobre él un fuerte impacto tal y como lo atestiguan los conocidos pasajes de la Crítica del Juicio en los que Kant hace uso del concepto de fuerza formativa. Esta fuerza formativa, que según el mismo Blumenbach se requería para darle forma propia al organismo o, en otras palabras, para dotar su crecimiento y desarrollo de una direccionalidad específica, conforme a su especie, y permitir en un momento dado su reproducción, se halla estrechamente vinculada con la idea de que el organismo posee un carácter con arreglo a fines. Pero para Kant se trata solamente de una idea reguladora y esa biología que es capaz de pensar en la naturaleza como algo que se organiza a sí mismo no es a fin de cuentas ninguna ciencia. Deja abierto, pues, un doble problema: el de la imposibilidad de conocer a las cosas en sí mismas, y el de la imposibilidad de una biología científica que tiene, al menos como uno de sus aspectos, el de la imposibilidad de dar cuenta de la epigénesis.

Schelling intenta osadamente resolver este doble problema de un solo golpe; propone que el sistema de la naturaleza y el del espíritu es el mismo, lo cual se explica porque el espíritu y la materia se hallan unidos originariamente. Pero esta misma unión explica también que la materia se organice a sí misma, pues la organización, el carácter con arreglo a fines de los seres vivos, sólo se puede entender en relación con un espíritu. Schelling requería de algo que organizara al ser vivo en el proceso epigenético, que le diera forma, estructura, intencionalidad, y ese algo no podía provenir desde afuera. Esto habría sido capitular ante el mecanicismo o ante la teología natural. La finalidad del organismo tenía que provenir de su interior y justamente este movimiento interno, constante, intencional, es lo que le proporciona su concepción de las fuerzas en continua oposición. Ya se trate del organismo individual en desarrollo, o de la naturaleza auto-construyéndose, el caso particular del problema que se le plantea a Schelling es en el fondo el de la epigénesis y la concepción dinámica de la materia su manera de resolverlo.

Con respecto al carácter de la teleología que Schelling invoca en esta concepción vale la pena subrayar un par de cosas. En primer lugar, pese al lenguaje exaltado que con frecuencia utiliza este autor, no me parece que estemos aquí ante una postura mística, esto es, una postura que enarbole, de acuerdo con la definición del DRAE (22a edición), una "doctrina religiosa y filosófica que enseña la comunicación inmediata y directa entre el hombre y la divinidad, en la visión intuitiva o en el éxtasis". La unidad entre materia y espíritu propuesta por Schelling, y la visión del organismo que la acompaña, rechaza ante todo la viabilidad de una concepción de las relaciones entre la naturaleza y el ser humano en la que una divinidad imparta direccionalidad y finalidad desde su trascendencia. El dios de Schelling es, en todo caso, un dios inmanente a la naturaleza que, paradójicamente para tratarse de 
una divinidad, deviene mundo orgánico e inorgánico en un proceso en el que las fuerzas fundamentales de la naturaleza juegan un papel central. Lo que Schelling niega en los pasajes que hemos examinado aquí, con mayor o menor fortuna, es la existencia del abismo insalvable entre sujeto y objeto que la epistemología clásica planteó desde sus inicios. La naturaleza es naturaleza sólo para nosotros y el sujeto y el objeto se hallan en todo caso en proceso continuo de auto-construcción.

En segundo lugar, esta postura está profundamente influida por la noción kantiana de organismo. Las características que Kant le adjudica al organismo, esto es, la interdependencia entre causa y efecto, la reivindicación de las causas finales en este terreno y el consecuente carácter con arreglo a fines del ser vivo, están todas ahí. Y, como Kant, Schelling se rehúsa a aceptar tanto el mecanicismo como el vitalismo; el concepto de fuerza vital es, para él, nulo en la medida en que una fuerza única, aislada, no puede existir por sí misma. Pero mientras que Kant afirmaba la incognoscibilidad de la realidad en sí, al margen de nosotros, y la imposibilidad de construir una ciencia de la vida, porque "la organización de la naturaleza no posee nada análogo a ninguna causalidad conocida [cognoscible] por nosotros" (Kant 1987, p. 255), Schelling apela a la inseparabilidad del espíritu y la naturaleza. Y una señal de esta inseparabilidad es para él, justamente, el carácter con arreglo a fines que los une. La naturaleza es resultado de un proceso epigenético, orientado a fines, durante el cual se va formando gradualmente, en virtud de fuerzas dinámicas y a partir de la materia. En este sentido, la teoría de la materia de la Naturphilosophie constituye la base de una visión organicista de la naturaleza que, sin ser vitalista, tampoco es mecanicista. No es mecanicista porque reconoce, ante todo, el carácter con arreglo a fines del ser vivo y su diferencia con una mera máquina. Pero tampoco es vitalista puesto que no "postula la existencia de un principio vital inmaterial considerado como la causa de los fenómenos que tienen que ver específicamente con la vida orgánica" (Lemaitre 2013, p. 7). La teleología de la que Schelling nos habla es inherente al mundo natural, inmanente a él, y es justamente este tipo de teleología lo que nos permite conocer a la naturaleza y lo que nos vincula a ella de manera íntima.

Bibliografía

Blumenbach, I.F. (1825), A Manual of the Elements of Natural History, London: W. Simpkin \& R. Marshall. (Traducción inglesa de R.T. Gore de la décima edición de: Handbuch der Naturgeschichte, Göttingen: in der Dieterichschen Buchhandlung, 1821.)

Gambaratto, A. (2014), “Teleology Beyond Regrets: On the Role of Schelling's Organicism in Treviranus' Biology”, Verifiche 43(1-3): 137-153.

Haller, A. (1752), "De partibus corporis humani sensibilibus et irritabilibus”, Commentarii Societatis Regiae Scientiarum Gottingensis 2: 114-158.

Gottfried, H.J. (1784), Outlines of a Philosophy of the History of Man, London: Bergman Publishers. (Traducción inglesa de T. Churchill de: Ideen zur Philosophie der Geschichte der Menschheit, Riga und Leipzig: Johann Friedrich Hartknoch, 1784.)

Huneman, P. (ed.) (2007), Understanding Purpose, New York: University of Rochester Press.

Kant, I. (1987), Critique of Judgment, Indianapolis/Cambridge: Hackett Publishing Company. (Traducción inglesa de Werner S. Pluhar de: Kritik der Urteilskraft, Berlin und Libau: Lagarde und Friedrich, 1790.)

Kant, I. (1785), Grundlegung zur Metaphysik der Sitten, Riga: Erstdruck.

Lemaitre, J.C. (2013), "Le statut de l'organisme dans la philosophie schellingienne de la nature", Klēsis Revue Philosophique 25: 3-36.

Leyte, A. (trad.) (1996), Escritos sobre filosofía de la naturaleza, Madrid: Alianza Universidad.

Newton, I. (1979), Opticks, New York: Dover Publications Inc.

Peterson, K.R. (2004), "Translator's Introduction”, en Schelling, F.W.J., First Outline of a System of the Philosophy of Nature, New York: State University of New York Press, pp. xi-xxxvii. 
Richards, R.J. (2002), The Romantic Conception of Life, Chicago/London: The University of Chicago Press.

Roe, S.A. (1981), Matter, Life and Generation, Cambrige: Cambridge University Press.

Schelling, F.W.J. (1797), “Introducción a ideas para una filosofía de la naturaleza”, en Leyte, A. (trad.) (1996), Escritos sobre filosofía de la naturaleza, Madrid: Alianza Universidad, pp. 69-117.

Schelling, F.W.J. (1799), "Introducción al Proyecto de un sistema de filosofía de la naturaleza o sobre el concepto de física especulativa y la organización interna de un sistema de esta ciencia”, en Leyte, A. (trad.) (1996), Escritos sobre filosofía de la naturaleza, Madrid: Alianza Universidad, pp. 119-174.

Schelling, F.W.J. (1798), "Von der Weltseele, eine Hypothese der höheren Physik zur Erklärung des allgemeinen Organismus”, en Schröter, M. (ed.), Schellings Werke, Erster Hauptband: Jugendschriften 1793-1798, München: C.H. Beck, 1927.

Sloan, P.R. (2002), "Preforming the Categories: Eighteenth-Century Generation Theory and the Biological Roots of Kant's A Priori", Journal of the History of Philosophy 40(2): 229-253. 